\title{
Stress classification based on human electromagnetic radiation analysis
}

\author{
Tengku ‘Afiah Mardhiah Tengku Zainul Akmal', Abd Hafiz Qayyum Abd Talib², Siti Zura A. Jalil', \\ Siti Armiza Mohd Aris ${ }^{4}$ \\ 1,2,3,4Razak Faculty of Technology and Informatics, Universiti Teknologi Malaysia (UTM), Malaysia \\ ${ }^{2}$ Cluster of Integrative Physiology and Molecular Medicine (CIPMM), Faculty of Medicine, Universiti Kuala Lumpur- \\ Royal College of Medicine Perak, Malaysia
}

\begin{tabular}{|c|c|}
\hline Article Info & ABSTRACT \\
\hline Article history: & Stress is a feeling of emotional or physical tension due to events that makes \\
\hline Received Oct 13, 2020 & $\begin{array}{l}\text { one feel frustrated, angry or nervous. It is a situation that trigger biological } \\
\text { response when a person encounters a threat or challenge. This paper }\end{array}$ \\
\hline Revised Mar 24, 2021 & discussed stress classification based on human electromagnetic radiation \\
\hline Accepted Mar 30, 2021 & $\begin{array}{l}\text { (EMR). EMR frequency are captured at seven major chakra points and being } \\
\text { analyzed using multivariate analysis of variance (MANOVA) to identify the }\end{array}$ \\
\hline Keywords: & $\begin{array}{l}\text { significance points for the classification. Locally weighted learning (LWL) } \\
\text { algorithm is used to classify the collected data. The results show stress }\end{array}$ \\
\hline $\begin{array}{l}\text { Biological response } \\
\text { Human electromagnetic }\end{array}$ & $\begin{array}{l}\text { classification using EMR based on third eye and throat chakra points } \\
\text { obtained accuracy of more than } 60 \% \text {. }\end{array}$ \\
\hline
\end{tabular}

Radiation

Stress classification

This is an open access article under the CC BY-SA license.

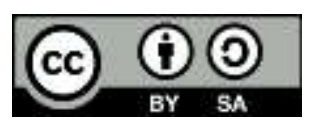

Corresponding Author:

Siti Zura A. Jalil

Razak Faculty of Technology and Informatics

Universiti Teknologi Malaysia

Jalan Sultan Yahya Petra, 54100 Kuala Lumpur, Malaysia

Email: sitizura.kl@utm.my

\section{INTRODUCTION}

Stress is defined as a threatened condition to the human body system stability. Human body reaction towards stress is known as adaptive process which involves physiological, biochemical and cognitive behavioral responses to gain body system stability [1]. Adaptive ability in dealing with stress will affect the risk of disease. Stress is a condition in which a person fails to adapt to the right conditions [2]. Stressful events are inevitable in life and need to overcome obstacles as a result of success. A person has the ability to control what they perceive as stress and how to respond to it. Stress response is the body's proactive step in adapting to a situation to encourage survival or motivate success, and can also be catastrophic when the body's response to stress is inappropriate. For instant, when a person experiences excessive situations or recurrent negative trauma, it can cause excessive stress levels and result in an inappropriate stress response that will prolong cortisol secretion [3], [4]. Stress has been a major concern in the current situations as chronic stress can leads to health issues such as heart disease, depression and anxiety. There are several factors that can trigger stress which includes major life changes, financial problems, and work. Many techniques have been used to assess stress. One of the current stress assessment is a lengthy process where patients need to answer multiple sets of questionnaires to be diagnose with stress [5], [6]. Several attempts also have been proposed on assessment and recognition of stress included using electrophysiosignal analysis 
[7], [8]. Therefore, this study proposed an alternative stress pre-assessment using human electromagnetic radiation (EMR) analysis.

Several evidences have shown the existence of energy field in the form of electric, magnetic, optical and acoustic emitted from and contained within the human body theoretically and experimentally [9]. Human energy field is defined as an extremely weak electromagnetic (EM) field but measurable EM that are formed from a collection of electromagnetic waves [10]. EM is produced around the human body due to the movement or rotation of particles. Cells, tissues and organs assemble molecules and each molecular interaction in the human body radiate unique energy spectrum. This spectrum is the EM radiation for each respective molecule [11], [12].

Alternative medicine philosophy such as chakra has practiced healing through energy field. It is mentioned that one's wellbeing is based on body energy balance and energy centered while any blockage or imbalance will affect the person's health condition. Chakra is derived from a Sanskrit word meaning 'wheel'. There are seven main chakras located from the perineum in the lower pelvis to the top of the head [13]. Each of these chakra points are associated with organs. When a person has an unresolved stress, this stress will cause disturbance to the body energy field which can contribute to physical illness. As the focus of this study is about stress, thus the chakra points that can be influenced by stress will be further discussed. There are several chakra points that are related to stress which are crown, third-eye, throat, solar plexus and root. Crown chakra is located at the top central of the skull. It is related to pineal gland which produces melatonin hormones for calming. Decreased melatonin production can cause anxiety or stress. Third eye chakra is located on the forehead between the eyebrows. This chakra externalizes the pineal gland thus treatment related to hormonal imbalance is done through this chakra as it governs lower brains, central nervous system, left eyes, ears and nose. Blockage of this chakra can lead to stress and anxiety [14]. Throat chakra is located in the throat to the base of the neck and collar bones. It is associated with communication and expression abilities. Weaken of this chakra can result to introvert behavior. Neck muscle tension due to stress is treated using acupuncture at this point. While throat is related to communication, solar plexus is associate to emotion which controls fear and anxiety. Thus, stress condition will inevitably influence the solar plexus chakra. It is located under the rib cage in the same area of diaphragm [15]. Root or base chakra is located at the base of the spine which is the chakra point that are closest to the earth. It responsible of anchoring the body on the physical plane and provide channel to express oneself. It is associated with adrenal medulla and cortex which produces adrenaline and cortisone. Blockage of this chakra will cause anxiety as the person is no longer grounded and reduced the gland secretion [14]. There were five chakra points that are relevant to stress, however the frequency of human EMR is captured at seven chakra points for this study. Further data analysis based on the frequency of human EMR is performed to confirm the points closely related to stress.

Previous study based on EMR frequencies analysis on gender classification shows that gender can be distinguished using k-nearest neighbour ( $\mathrm{kNN}$ ) classification method [16]. There are 13 out of 23 points that are significant to differentiate gender. Male and female have different distribution of frequency radiations. Males are observed to have higher range of frequencies on both left and right side of the body compare to females [17]. Several studies on human EMR also demonstrates significant result in classifying body segment on upper body, torso, arm and lower body [18], and on left, right and chakra [19]. In addition, studies shown that EMR can be classified based on the person's health condition [20], [21]. Significant results have been shown for down-syndrome and non-down syndrome person [20] and for stroke patients and to non-stroke participants, which support the assumption of human conditions can affect human EMR [21].

A recent comparative study shows that kNN classifier gives the lowest accuracy when comparing with J48, Bayes Net and locally weighted learning (LWL) algorithm in predicting breast cancer survival rate despite the high performance of kNN in previous studies. The LWL algorithm produces highest accuracy with $66.2 \%$ while $\mathrm{kNN}$ only able to predict at $56.1 \%$ accuracy [22]. The LWL performance also surpass kNN in identifying defective software modules using imbalanced dataset with $92.23 \%$ accuracy when validated with 10 -fold cross validation and $91.08 \%$ accuracy when tested with percentage split of $66 \%$. The result also has been validated using paired t-test with $99 \%$ confidence level [23]. Thus in this study, LWL algorithm will be used to classify the stress.

\section{METHODOLOGY}

The proposed study involved four steps which are data acquisition, statistical analysis for preprocessing, classification and validation. Further details about these steps are explained in the next subsections. 


\subsection{Sampling}

The subjects for this study are students from Universiti Teknologi Malaysia, Kuala Lumpur. There are forty (40) volunteer students consist of twenty-nine (29) males and eleven (11) females are involves in this study. The stress is induced through virtual reality (VR) technology [24], [25]. The EMR frequencies are collected two (2) times, i.e before the stress is induced (before VR session) and after the stress is induced (after VR session). During data collection, the measurement room temperature is set constant at $24^{\circ} \mathrm{C}$ and the data collection session is limited to four sessions per day. Session 1 is from 9.00am to 10.00am, session 2 is from $10.30 \mathrm{am}$ to $11.30 \mathrm{am}$, session 3 is from $2.00 \mathrm{pm}$ to $3.00 \mathrm{pm}$ and session 4 is from $3.30 \mathrm{pm}$ to $4.30 \mathrm{pm}$.

\subsection{EMR data collection}

The EMR data is collected at seven points using body radiation wave detector which are the crown, third eye, throat, heart, solar plexus, sacral and root chakra points. The points' label and location of each chakra points are described in Table 1. The EMR data acquisition is performed as shown in Figure 1. The EMR readings are taken five times at each point. The average EMR reading for each chakra points are calculated before further analysis.

Table 1. Location of chakra points

\begin{tabular}{ccl}
\hline Chakra Points & Label & \multicolumn{1}{c}{ Location } \\
\hline Crown & CA & Top of the head \\
Third Eye & CB & Forehead between the eyes \\
Throat & CC & Throat \\
Heart & CD & Centre of chest just above the heart \\
Solar Plexus & CE & Upper abdomen in the stomach area \\
Sacral & CF & Lower abdomen, about two inches below the navel \\
Root & CG & Base of spine in tailbone area \\
\hline
\end{tabular}
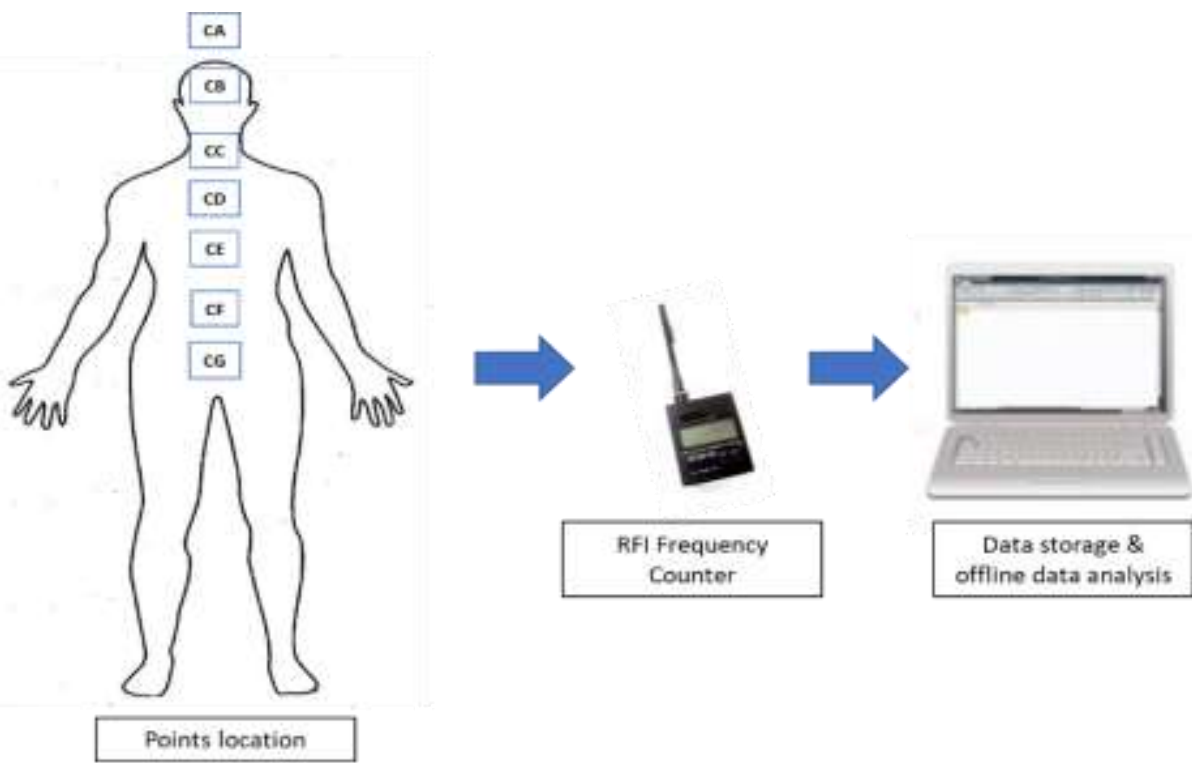

Figure 1. EMR data acquisition

\subsection{Statistical analysis}

The collected EMR data is analyzed using multivariate analysis of variance (MANOVA) in SPSS Statistics software version 23 to find significant points for the classification of stress state. MANOVA is a generalization of the general linear model of statistical analysis to situations where there are multiple dependent measures. In this study, the independent measures are VR stress session (before and after the stress is induced) and the dependent measures are EMR readings on chakra points of CA to CG. The analysis results in the F-ratio statistic is calculated for the EMR readings to indicate whether different values of before and after VR stress session have statistically significant effect on the EMR readings on chakra points. MANOVA are based on F-test, the larger F value and the smaller $p$-value (less than 0.05 for the sig. value). Hence, the $\mathrm{F}$ value and $\mathrm{P}$ value is evaluated to identify which points are significant in identifying the stress. 


\subsection{Data classification}

The datasets with significant attributes are classified using waikato environment for knowledge analysis (WEKA) software tool by utilizing the locally weighted learning (LWL) classifier. LWL classifier is one of the lazy learner classifiers. Lazy learners are advantageous when performing prediction using single training sets because only the immediate sections of the instance space are occupied by objects to be classified will be modeled. It can improve prediction accuracy by allowing the system to focus on deriving possible decision for exact points of the instance space for prediction. LWL refers to supervised learning of continuous functions which are in the context of kernel regression [26]. LWL forms lazy model around a point of interest whereby only training data that is local to that point is used during classification. LWL can be observed as approximation method function [27]. It is formulated as:

$$
F\left(x_{q}\right)=w_{0}+w_{1} a_{1}\left(x_{q}\right)+\cdots+w_{n} a_{n}\left(x_{q}\right)
$$

Where $a_{i}\left(x_{q}\right)$ is the $i^{t h}$ attributes of point $x_{q}, w_{i}$ is the coefficient for each $a_{i}(x)$ and $F(x)$ is the target function determined by $a_{i}(x)$ and $w_{i}$. The lazy model is used to fit nearby data points by defining the error criterion as expressed in (2):

$$
E\left(x_{q}\right)=\frac{1}{2} \sum_{x \in D}(F(x)-f(x))^{2} K\left(\frac{d\left(x_{q}, x\right)}{\beta}\right)
$$

Where $x_{q}$ is the query point, the data point set containing k-nearest data point. $K$ is the kernel function to calculate weight for each data point to the distance. While $d\left(x_{q}, x\right)$ is the distance between query point $x_{q}$ to each data point $x$. The favorable approximation for the function output $F(x)$ can be obtained by determining the $w_{i}$. Gradient descent method is used to get the best estimation of $w_{i}$ with minimal error criterion $\left(x_{q}\right)$. The training criterion is formulated as:

$$
\Delta w_{i}=\eta \sum_{x \in D} K\left(\frac{d\left(x_{q}, x\right)}{\beta}\right)(F(x)-f(x)) a_{i}(x)
$$

Where $\eta$ is the learning rate and $\beta$ is the bandwidth. The new weight is obtained through as (4):

$$
w_{i}=w_{i}+\Delta w_{i}
$$

\subsection{Data validation}

The classification training set is validated using k-fold cross validation. Cross validation is used to evaluate the accuracy of the classifier by repeating the classification process based on define numbers of $k$. The initial dataset will be randomly partition into $k$ mutually exclusive or also known as folds, $D_{1}, D_{2},::: D_{k}$, each of approximately equal size. The training and testing is executed for $k$ times. For each iteration $i$, partition $D_{i}$ will be used as test set while the remaining partitions are used to train the model. The accuracy estimate is based on the overall number of correct classifications from $k$ iterations and divided by total number of tuples in the initial data [28].

The classification evaluation are measured by true positive (TP) which refer to positive tuples that were correctly labeled by the classifier, true negative (TN) which is the negative tuples that were correctly labeled by the classifier, false positive (FP) are the negative tuples that were incorrectly classified and false negative $(\mathrm{FN})$ are the positive tuples that were incorrecly labeled by the classifier.

\section{RESULTS}

Table 2 shows the result of MANOVA analysis for the overall data. The results demonstrate that throat chakra is the most significant to differentiate stress state as the sig. or $p$-value is less than 0.05 , then followed by third eye and solar plexus chakra. The overall classification results by each chakra points are tabulated in Table 3. The third eye chakra has the highest accuracy with $65 \%$ correctly classified and $90 \% \mathrm{TP}$ Rate for classification of before VR session and 40\% TP Rate for after VR session. The second highest correctly classified accuracy is the throat chakra with $53.80 \%$ and followed by root chakra with $52.50 \%$.

The overall classification results by combination of multiple chakra points are displayed in Table 4. Combination of chakra points are based on significant points found in the statistical analysis. The combination of third eye (CB) and throat chakra (CC) produce a highest accuracy of classification up to $66.25 \%$ and $90 \%$ of TP rate for before VR session. The second highest correctly classified accuracy is 
followed by combination of crown (CA), third eye (CB), throat (CC), sacral (CF) and root (CG) with $65 \%$ accuracy.

Table 2. MANOVA analysis for overall data

\begin{tabular}{cccc}
\hline Chakra & Points & F & Sig. $(p$-value $)$ \\
\hline Crown & CA & 1.839 & 0.179 \\
Third Eye & CB & 3.883 & 0.052 \\
Throat & CC & 6.881 & 0.011 \\
Heart & CD & 2.007 & 0.161 \\
Solar Plexus & CE & 3.586 & 0.062 \\
Sacral & CF & 1.684 & 0.198 \\
Root & CG & 1.414 & 0.238 \\
\hline
\end{tabular}

Table 3. Classification of chakra points

\begin{tabular}{cccccc}
\hline Chakra & Points & Correctly Classified & Incorrectly Classified & $\begin{array}{c}\text { TP Rate } \\
\text { (After VR session) }\end{array}$ & $\begin{array}{c}\text { TP Rate } \\
\text { (Before VR session) }\end{array}$ \\
\hline Crown & CA & 50.00 & 50.00 & 0.300 & 0.700 \\
Third Eye & CB & 65.00 & 35.00 & 0.400 & 0.900 \\
Throat & CC & 53.80 & 46.20 & 0.200 & 0.900 \\
Heart & CD & 42.50 & 58.50 & 0.200 & 0.600 \\
Solar Plexus & CE & 43.80 & 56.20 & 0.300 & 0.400 \\
Sacral & CF & 46.30 & 54.70 & 0.400 & 0.600 \\
Root & CG & 52.50 & 48.50 & 0.500 & 0.500 \\
\hline
\end{tabular}

Table 4. Classification of combination chakra points

\begin{tabular}{ccccccccccc}
\hline CA & CB & CC & CD & CE & CF & CG & $\begin{array}{c}\text { Correctly } \\
\text { Classified }\end{array}$ & $\begin{array}{c}\text { Incorrectly } \\
\text { Classified }\end{array}$ & $\begin{array}{c}\text { TP Rate } \\
\text { (After VR session) }\end{array}$ & $\begin{array}{c}\text { TP Rate } \\
\text { (Before VR session) }\end{array}$ \\
\hline$\sqrt{ }$ & $\sqrt{ }$ & $\sqrt{ }$ & $\sqrt{ }$ & $\sqrt{ }$ & $\sqrt{ }$ & $\sqrt{ }$ & 63.75 & 36.25 & 0.400 & 0.875 \\
$\sqrt{ }$ & $\sqrt{ }$ & $\sqrt{ }$ & - & $\sqrt{ }$ & $\sqrt{ }$ & $\sqrt{ }$ & 63.75 & 36.25 & 0.400 & 0.875 \\
$\sqrt{ }$ & $\sqrt{ }$ & $\sqrt{ }$ & - & - & $\sqrt{ }$ & $\sqrt{ }$ & 65.00 & 35.00 & 0.400 & 0.900 \\
$\sqrt{ }$ & $\sqrt{ }$ & $\sqrt{ }$ & - & - & - & $\sqrt{ }$ & 62.25 & 37.75 & 0.375 & 0.850 \\
- & $\sqrt{ }$ & $\sqrt{ }$ & - & - & - & $\sqrt{ }$ & 62.50 & 37.50 & 0.375 & 0.875 \\
- & $\sqrt{ }$ & $\sqrt{ }$ & - & - & - & - & 66.25 & 33.75 & 0.425 & 0.900 \\
\hline
\end{tabular}

\subsection{EMR stress data}

The result of MANOVA analysis for EMR stress subject's shows no significant difference between before and after VR stress session. The chakra points that are close to significant are solar plexus, throat, heart, third eye and sacral as shown in Table 5. The classification result for stress subject's is shown in Table 6. The results indicate throat chakra has the highest classification accuracy of $63.64 \%$ with $100 \% \mathrm{TP}$ rate for before VR stress session. The next highest accuracy is sacral chakra with $59.09 \%$ and solar plexus chakra with $54.55 \%$ accuracy.

In Table 7, the combination of throat (CC), solar plexus (CE) and sacral (CF), and combination of throat (CC) and sacral chakra (CF) produces a highest accuracy of correctly classified up to $59.09 \%$. The combination of throat and sacral chakra yields a highest TP rate up to $90.9 \%$ for classification before VR session. Meanwhile, combination of throat, solar plexus and sacral chakra produces slightly lower TP rate at $81.8 \%$. This finding is consistent with previous studies showing the relation of several chakra points to stress [19].

Table 5. MANOVA analysis for stress subjects

\begin{tabular}{cccc}
\hline Chakra & Points & F & Sig. $(p$-value $)$ \\
\hline Crown & CA & 0.565 & 0.461 \\
Third Eye & CB & 1.931 & 0.180 \\
Throat & CC & 2.475 & 0.131 \\
Heart & CD & 2.048 & 0.168 \\
Solar Plexus & CE & 4.147 & 0.055 \\
Sacral & CF & 1.655 & 0.213 \\
Root & CG & 0.64 & 0.433 \\
\hline
\end{tabular}


Table 6. Stress subjects classification by chakra points

\begin{tabular}{cccccc}
\hline Chakra & Points & Correctly Classified & Incorrectly Classified & $\begin{array}{c}\text { TP Rate } \\
\text { (After VR session) }\end{array}$ & $\begin{array}{c}\text { TP Rate } \\
\text { (Before VR session) }\end{array}$ \\
\hline Crown & CA & 40.91 & 59.09 & 0.273 & 0.545 \\
Third Eye & CB & 45.45 & 54.55 & 0.545 & 0.364 \\
Throat & CC & 63.64 & 36.36 & 0.273 & 1.00 \\
Heart & CD & 50.00 & 50.00 & 0.273 & 0.727 \\
Solar Plexus & CE & 54.55 & 45.45 & 0.364 & 0.727 \\
Sacral & CF & 59.09 & 40.91 & 0.364 & 0.818 \\
Root & CG & 50.00 & 50.00 & 0.636 & 0.364 \\
\hline
\end{tabular}

Table 7. Stress subjects classification by combination of chakra points

\begin{tabular}{ccccccccccc}
\hline CA & CB & CC & CD & CE & CF & CG & $\begin{array}{c}\text { Correctly } \\
\text { Classified }\end{array}$ & $\begin{array}{c}\text { Incorrectly } \\
\text { Classified }\end{array}$ & $\begin{array}{c}\text { TP Rate } \\
\text { (After VR session) }\end{array}$ & $\begin{array}{c}\text { TP Rate } \\
\text { (Before VR session) }\end{array}$ \\
\hline$\sqrt{ }$ & $\sqrt{ }$ & $\sqrt{ }$ & $\sqrt{ }$ & $\sqrt{ }$ & $\sqrt{ }$ & $\sqrt{ }$ & 36.36 & 63.64 & 0.273 & 0.455 \\
- & $\sqrt{ }$ & $\sqrt{ }$ & $\sqrt{ }$ & $\sqrt{ }$ & $\sqrt{ }$ & $\sqrt{ }$ & 40.91 & 59.09 & 0.273 & 0.545 \\
- & - & $\sqrt{ }$ & $\sqrt{ }$ & $\sqrt{ }$ & $\sqrt{ }$ & $\sqrt{ }$ & 40.91 & 59.09 & 0.273 & 0.545 \\
- & - & $\sqrt{ }$ & - & $\sqrt{ }$ & $\sqrt{ }$ & $\sqrt{ }$ & 50.00 & 50.00 & 0.273 & 0.727 \\
- & - & $\sqrt{ }$ & - & $\sqrt{ }$ & $\sqrt{ }$ & - & 59.09 & 40.91 & 0.364 & 0.818 \\
- & - & $\sqrt{ }$ & - & - & $\sqrt{ }$ & - & 59.09 & 40.91 & 0.273 & 0.909 \\
\hline
\end{tabular}

\subsection{EMR result based on subjects' feedback}

Based on the subjects' feedback on stress after the VR stress session experiment, majority of the subjects shows doesn't feel stress. Figure 2 illustrates the finding. There is only $19 \%$ of the male subjects and $43 \%$ of female subjects were experienced stress after VR stress session, giving a total of 11 subjects out of 40 subjects. From the findings, it demonstrates gender differences on stress experiences in which female more susceptible of developing stress when exposed to physiological stress as compared to male. This finding is in line to previous studies [6], [8]. Studies also shows that females have slower rate of adapting to virtual reality environment compared to the males, indicating that VR or any type of stressor will give higher impact in females with compared to males [29], [30].

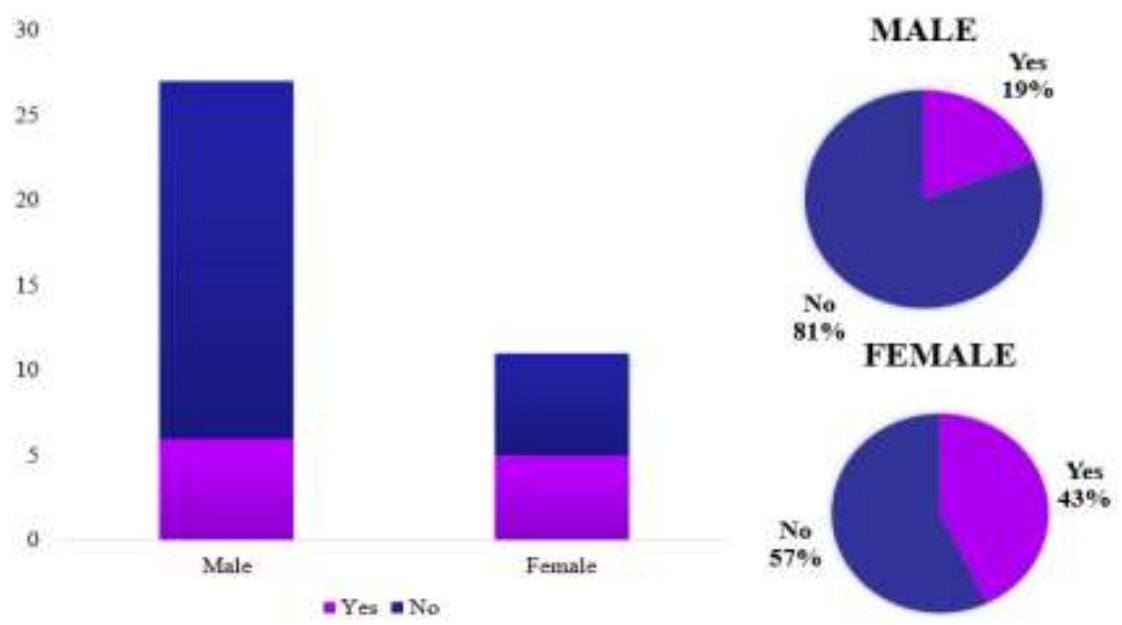

Figure 2. Post VR experience feedback on stress

\section{CONCLUSION}

Stress has been a major concern in the current situations as chronic stress can leads to health issues such as heart disease, depression and anxiety. This paper discussed stress classification based on human electromagnetic radiation (EMR) analysis. There are twenty-nine males and eleven female's student involved in this study. The human EMR data are analyse using statistical analysis of MANOVA and classified using LWL algorithm. Based on the overall EMR dataset, the finding shows that throat is the most significant point and followed by third eye. The result shows that the classification accuracy of this combinations is more than $60 \%$ accuracy. This finding in line with the previous studies indicating the relation of several chakra points to 
stress. However, since the number of subjects are unbalanced with the majority of the subjects are males, and based on the subjects' feedback on stress after the VR stress session, most of male subject's did not feel stressed while using VR. This factor may contribute to the percentage accuracy obtained of correctly classify. Although this study is capable of classifying stress using human EMR, further investigation with more and balance number of subject will be performed to distinguish and classify the human EMR on stress. In addition, this study will be associate with a well-established bio feedback instruments on stress identification such as electroencephalogram (EEG) for future research.

\section{ACKNOWLEDGEMENTS}

The authors wish to express their appreciation to Universiti Teknologi Malaysia for supporting the research. This work is supported in part by Ministry of Higher Education under Fundamental Research Grant Scheme (FRGS/1/2018/ICT04/UTM/03/3).

\section{REFERENCES}

[1] M. Stults-Kolehmainen and R. Sinha, "The Effects of Stress on Physical Activity and Exercise," Sports medicine (Auckland, N.Z.), vol. 44, no. 1, pp. 81-121, 2013, doi: 10.1007/s40279-013-0090-5.

[2] N. H. A. Hamid, N. Sulaiman, S. A. M. Aris, Z. H. Murat, and M. N. Taib, "Evaluation of human stress using EEG Power Spectrum," in 6th International Colloquium on Signal Processing \& its Applications, 2010, pp. 1-4, doi: 10.1109/CSPA.2010.5545282.

[3] K. Hannibal and M. Bishop, "Chronic Stress, Cortisol Dysfunction, and Pain: A Psychoneuroendocrine Rationale for Stress Management in Pain Rehabilitation," Physical therapy, vol. 94, no. 12, pp. 1816-1825, 2014, doi: 10.2522/ptj.20130597.

[4] H. Yaribeygi, Y. Panahi, H. Sahraei, T. P. Johnston, and A. Sahebkar, "The impact of stress on body function: A review," (in eng), EXCLI journal, vol. 16, pp. 1057-1072, 2017, doi: 10.17179/excli2017-480.

[5] Sandhu, Sukhvinder Singh et al. "The Malay Version of the Perceived Stress Scale (PSS)-10 is a Reliable and Valid Measure for Stress among Nurses in Malaysia," The Malaysian journal of medical sciences : MJMS vol. 22, no. 6, 2015, pp. 26-31.

[6] A. Baharum, S. Mea Tanalol, C. Jian, M. Omar, N. A. Mat Noor, and N. Yusop, "Stress catcher application for mobile stress monitoring using questionnaire-based," Indonesian Journal of Electrical Engineering and Computer Science, vol. 16, no. 2, p. 917-924, 2019, doi: 10.11591/ijeecs.v16.i2.pp917-924.

[7] N. Halim, K. Sidek, and H. Mansor, "Stress Recognition Using Photoplethysmogram Signal," Indonesian Journal of Electrical Engineering and Computer Science, vol. 8, no. 2, pp. 495-501, 2017, doi: 10.11591/ijeecs.v8.i2.pp495-501.

[8] N. S. Nor Shahrudin, K. A. Sidek, and A. Z. Jusoh, "Electrocardiogram (ECG) based stress recognition integrated with different classification of age and gender," Indonesian Journal of Electrical Engineering and Computer Science, vol. 15, no. 1, pp. 199-210, 2019, doi: 10.11591/ijeecs.v15.i1.pp199-210.

[9] G. Rein, "Bioinformation Within the Biofield: Beyond Bioelectromagnetics," The Journal of Alternative and Complementary Medicine, vol. 10, no. 1, pp. 59-68, 2004, doi: 10.1089/107555304322848968.

[10] J. L. Oschman, "Science and The Human Energy Field," Reiki News Magazine, vol. 1, no. 3, pp. 27-44, 2002.

[11] Z. Movaffaghi and M. Farsi, "Biofield threrapies : Biophysical basis and biological regulations?," Complementary Therapies in Clinical Practice, vol. 15, no. 1, pp. 35-37, 2009, doi: 10.1016/j.ctcp.2008.07.001.

[12] B. Rubik, D. Muehsam, R. Hammerschlag, and S. Jain, "Biofield Science and Healing: History, Terminology, and Concepts," (in eng), Global advances in health and medicine, vol. 4, no. Suppl, pp. 8-14, 2015, doi: 10.7453/gahmj.2015.038.suppl.

[13] A. K. Gilmore et al., "Gender differences in subjective stress and neuroendocrine response to a stress task among individuals with opioid dependence: A pilot study," Addictive Behaviors, vol. 92, pp. 148-154, 2019, doi: 10.1016/j.addbeh.2018.12.022.

[14] S. Shienfield, "The Art of Chakra Balancing", Andrews McMeel Publishing, 2005.

[15] J. R. Cross, N. Ellis, and J. Amaro, "Acupuncture and the Chakra Energy System: Treating the Cause of Disease", North Atlantic Books, 2012.

[16] S. Z. A. Jalil, M. N. Taib, H. A. Idris, and M. M. Yunus, "Gender Classification Based on Human Radiation Wave Analysis," in UkSim 13th International Conference on Computer Modelling and Simulation, 2011, pp. 59-63, doi: 10.1109/UKSIM.2011.21.

[17] S. Z. A. Jalil, M. N. Taib, H. Abdullah, and M. M. Yunus, "Frequency Radiation Characteristic Around the Human Body," International Journal of Simulation: Systems, Science and Technology, vol. 12, no. 1, pp. 35-39, 2011, doi: 10.5013/IJSSST.a.12.01.05.

[18] S. Z. A. Jalil, S. A. M. Aris, N. A. Bani, H. M. Kaidi, and M. N. Muhtazaruddin, "Recognition of body segment based on human electromagnetic radiation analysis," in IEEE EMBS Conference on Biomedical Engineering and Sciences, 2016, pp. 571-576, doi: 10.1109/IECBES.2016.7843514.

[19] S. Z. A. Jalil, S. A. M. Aris, N. A. Bani, M. N. Muhtazaruddin, and S. Usman, "Segmentation of the human body based on frequency of human electromagnetic radiation," in Indonesian Journal of Electrical Engineering and Computer Science, vol. 18, no. 1, pp. 268-275, 2020, doi: 10.11591/ijeecs.v18.i1.pp268-275. 
[20] M. Rosdi, R. S. S. Abd Kadir, Z. Hj Murat and N. Kamaruzaman, "The comparison of human body Electromagnetic radiation between Down Syndrome and Non Down Syndrome person for brain, chakra and energy field stability score analysis," IEEE Control and System Graduate Research Colloquium, Shah Alam, Selangor, 2012, pp. 370-375, doi: 10.1109/ICSGRC.2012.6287194.

[21] R. S. S. A. Kadir, Z. H. Murat, M. N. Taib, and S. Z. A. Jalil, "The characteristics of human body electromagnetic radiation frequencies for stroke patients and non-stroke participants," in IEEE Symposium on Computer Applications \& Industrial Electronics (ISCAIE), 2017, pp. 212-216, doi: 10.1109/ISCAIE.2017.8074979.

[22] Y. Cao, and X. Zhang, "Research on Data Mining Method for Breast Cancer Case Data," Sun X., Pan Z., Bertino E. (eds) Cloud Computing and Security. ICCCS 2018. Lecture Notes in Computer Science, vol 11064. Springer, Cham, pp. 71-78, 2018.

[23] J. Ge, J. Liu, and W. Liu, "Comparative Study on Defect Prediction Algorithms of Supervised Learning Software Based on Imbalanced Classification Data Sets," in 19th IEEE/ACIS International Conference on Software Engineering, Artificial Intelligence, Networking and Parallel/Distributed Computing (SNPD), 2018, pp. 399-406, doi: 10.1109/SNPD.2018.8441143.

[24] T. Finseth, N. Barnett, E. Shirtcliff, M. Dorneich, and N. Keren, "Stress Inducing Demands in Virtual Environments," Proceedings of the Human Factors and Ergonomics Society Annual Meeting, vol. 62, no. 1, pp. 2066-2070, 2018, doi: 10.1177/1541931218621466.

[25] J. Santl, S. Youssef, A. Plab, S. Wüst, B. Kudielka, and A. Mühlberger, "Gender Differences in Stress Responses during a Virtual Reality Trier Social Stress Test," International Journal of Virtual Reality, vol. 19, no. 2, pp. 2-15, 2019, doi: 10.20870/IJVR.2019.19.2.2912.

[26] J.-A. Ting, S. Vijayakumar, and S. Schaal, "Locally Weighted Regression for Control," C. Sammut \& G. I. Webb (Eds.), Encyclopedia of Machine Learning. Boston, MA: Springer US, vol. 11, pp. 613-624, 2010, doi: 10.1007/978-1-4899-7502-7_493-1.

[27] C.-C. Wei, "Comparing lazy and eager learning models for water level forecasting in river-reservoir basins of inundation regions," Environmental Modelling \& Software, vol. 63, pp. 137-155, 2015, doi: 10.1016/j.envsoft.2014.09.026.

[28] J. Han, M. Kamber, and J. Pei, “Classification: Basic Concepts,” J. Han, M. Kamber, \& J. Pei (Eds.), Data Mining (Third Edition), Boston: Morgan Kaufmann, pp. 327-391, 2012.

[29] M. Wei, J. Luo, H. Luo, and R. Song, "The effect of gender on vection perception and postural responses induced by immersive virtual rotation drum," in 8th International IEEE/EMBS Conference on Neural Engineering (NER), 2017, pp. 473-476, doi: 10.1109/NER.2017.8008392.

[30] P.-A. Fransson et al., "Postural instability in an immersive Virtual Reality adapts with repetition and includes directional and gender specific effects," Scientific Reports, vol. 9, no. 1, pp. 1-10, 2019, doi: 10.1038/s41598-01939104-6.

\section{BIOGRAPHIES OF AUTHORS}
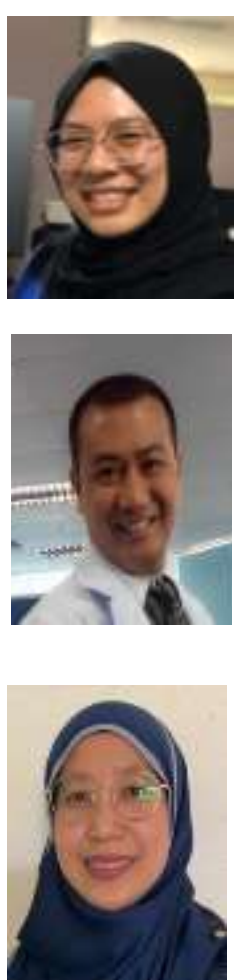

Tengku ‘Afiah Mardhiah Tengku Zainul Akmal was born in Malaysia, on 28 May, 1990. She received Bachelor of Aircraft Eng. Tech.(Hons) Mechanical from Universiti Kuala Lumpur (UniKL) and MSc in Systems Engineering from Universiti Teknologi Malaysia (UTM) in 2009 and 2019 respectively. She has been working on image classification and bio-signals analysis during her Masters' studies. She is currently a data scientist in NXP Semiconductors and her present work are related with image processing, pattern recognition and anomalous signals detection in various semiconductor testing areas.

Abd Hafiz Qayyum Abd Talib was born in Malaysia, on 11 September, 1985. He received Bachelor Degree in Bachelor of Aircraft Engineering Technology (Hons.) Mechanical from Universiti Kuala Lumpur Malaysian Institute of Aviation Technology (UniKL MIAT) and Master of Medical Science (Physiology) from Universiti Kuala Lumpur Royal Collage of Medicine Perak (UniKL RCMP). He is currently pursuing his PhD degree level in Electrical and Electronic Engineering (Biomedical Engineering) at Universiti Teknologi Malaysia (UTM) Kuala Lumpur. He involved many research work related with Electrical Power, Biomedical Engineering, Integrative Physiology and Molecular Medicine including Electrophysiology, Human Gaits, Artificial Intelligence (AI) and Internet of Things (IoT).

Siti Zura A. Jalil was born in Malaysia, on 31 August, 1975. She received a B. Eng. (Hons) degree and M. Eng. degree in Electrical Engineering from Universiti Technologi Malaysia in 1998 and 2001, respectively, and the PhD Degree in Biomedical Engineering in 2014 from Universiti Teknologi MARA, Malaysia. She is a senior lecturer at Razak Faculty of Technology and Informatics, Universiti Teknologi Malaysia, Kuala Lumpur, Malaysia. She has led research in advanced signal processing and data analytics with applications in biomedical engineering particularly in human electromagnetic radiation and bio-signal analysis. She is also interested in the field of biomechanic engineering of gait studies. She is member to IEEE Malaysia chapters of Signal Processing Society, and Engineering in Medicine and Biology Society. 


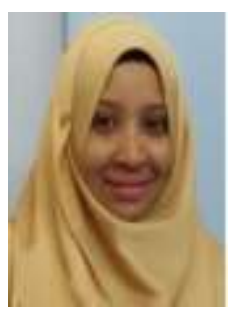

Siti Armiza Mohd Aris was born in Malaysia, on 11 September, 1975. She received the B.Eng degree in Electrical Engineering (Microelectronics) from Universiti Teknologi Malaysia in 1998, and the M.Eng. as well as Ph.D degrees in Electrical Engineering from Universiti Teknologi Malaysia in 2001 and Universiti Teknologi MARA in 2016 respectively. She started as a tutor in 1998 and now has become a senior lecturer at Universiti Teknologi Malaysia, Kuala Lumpur. In 2012, she joined the UTM Razak School of Engineering and Advanced Technology as a Lecturer and Researcher, a school that offers undergraduate and postgraduate students from various disciplines. Her current research interests include EEG signal processing, bio-signal processing, psycho-physiological interactive tools, and bio-signal monitoring tools. She is a member of IEEE Malaysia Section, IEEE EMBS Malaysia Chapter and IEEE Signal Processing Society Malaysia Chapter. In 2016, her research paper has been recognised by the IEEE WIE and awarded as the best research paper for her outstanding work. 